\title{
Katholische charismatische Bewegung in Weißrussland: Mechanismen der Entstehung und Tendenzen der Entwicklung
}

\author{
MARYNA KAZMIRUK*
}

The purpose of the paper is a critical analysis of the charismatic movement as an example of ecstatic groups of renewal in the Catholic Church in Belarus. During the analysis of this phenomenon in Belarus in example of charismatic communities of ecstatic orientation were uncovered phase formation and growth of charismatic groups, as well as the characteristic features of the development of Catholic "renewal movement" on the territory of Belarus. The paper presents the analytical data of contemporary religious situation that obtained qualitative research methods. They reflect popular trends observed among the followers of the Catholic charismatic communities ecstatic and modern processes that occur in groups.

Keywords: Roman-catholic Church, catholic charismatic renewal, charismatic groups, the Holy Spirit, glossolalia, ecstatic groups, Pentecostalism, catholic Pentecostalism

\section{Einführung}

Die neueste Zeit wird durch Innovationsprozesse gekennzeichnet, die auf eine tiefgehende Transformation des gesellschaftlichen Lebens hindeuten. Der konfessionelle Bereich ist voll in diesen Prozess einbezogen, was seinen Ausdruck in der Dynamik sowohl von traditionellen als auch nicht traditionellen Formen der Religiosität in der modernen Gesellschaft findet.

Diese Veröffentlichung lenkt die Aufmerksamkeit darauf, wie wichtig es ist, die Rolle der nicht traditionellen Religiosität in der Dynamik der modernen Kultur zu berücksichtigen und die dabei beobachteten Formen und Tendenzen innerhalb der traditionellen Weltreligionen, konkret im Rahmen der katholischen Tradition, zu identifizieren und zu klassifizieren.

Die Analysen des folgenden Artikels sollen einige Aspekte der kulturell-historischen Dynamik der katholischen charismatischen Bewegung beleuchten; diese Angaben beruhen auf empirischen Daten, die unter Anwendung von qualitativen Beobachtungsmethoden gesammelt wurden. Das durchgeführte Insiding wurde mit unstrukturierten Interviews mit Adepten

* Maryna Kazmiruk, Doktorand am Institut für Kulturwissenschaft an der Belarussischen Staatlichen Universität für Kultur und Kunst. Adress: 4 Radiatornyj Gasse, 7, 220093, Minsk, Belarus; e-mail: cincinnata@mail.ru. 
während der nichtoffiziellen Treffen, mit Audio- und Videoaufnahmen aus dem persönlichen Archiv der

Autorin sowie mit Materialien, die als gruppeninterne Literatur identifiziert werden können, wesentlich vervollständigt.

Sich stützend auf das empirische Material, das im Laufe der Beobachtungen gesammelt wurde, wurde eine Analyse von Transformationen, die in Gruppen der katholischen charismatischen Bewegung in Weißrussland stattgefunden haben, durchgeführt. Die Aufnahme von mystischen Formen der religiösen Erfahrung durch Adepten der charismatischen katholischen Gruppen, ähnlich der Erfahrung, die in den Praktiken von modernen mystischen Strömungen vorzufinden ist, bedeutet eine Abweichung von zentralen Elementen des Katholizismus. Außerdem ist zu bedenken, dass ekstatische Erfahrungen der Wiedergeburt, wie sie in einigen Gruppen zu beobachten sind, auf eine Spiritualität hinweisen, die Aspekten des Übergangs von den traditionellen Religionen zu neuen religiösen Bewegungen ähnlich ist. ${ }^{1}$ Die katholische Pfingstbewegung ist in der Missionstätigkeit unter der Bevölkerung der Mittelschicht und unter den gut ausgebildeten traditionellen Gläubigen aktiv. ${ }^{2}$ Der Religiositätstyp, der in den charismatischen Gruppen zu finden ist, unterscheidet sich wesentlich - wenigstens für Katholiken - von dem Typ, in welchen sie integriert wurden. Die Wiederbelebung von einigen Formen der religiösen Erfahrung (wie zum Beispiel Glossolalie) wird mit der Wiedergeburt des Glaubens - „Revivalism“ in der römisch-katholischen Kirche - identifiziert. Zur Begründung der Legitimität der verwendeten Praktiken appellieren die Adepten an die „Zeiten der Urkirche“ und setzen sich mit der „apostolischen Bewegung unserer Tage“ gleich. ${ }^{3}$ Als Folge davon ist eine Veränderung der Dogmatik (der grundlegenden Fragen der Trinitätstheologie und der Christologie) zu beobachten, während die sekundären Dogmen (wie zum Beispiel der Primat des Papstes) keinen Transformationen ausgesetzt sind. ${ }^{4}$

Die analysierten katholischen charismatischen Gruppen, insbesondere diejenigen, die sich an der Peripherie befinden und damit einer geringeren Kontrolle unterliegen, können Orte des Übergangs vom Katholizismus zu

1 Peter Halama, Júlia Halamová, „Process of religious conversion in the Catholic Charismatic movement: A qualitative analysis" in: Archive for the Psychology of Religion 27 (1/2005), S. 69-92.

\section{Ibidem.}

3 Kilian McDonnell, "Charismatic renewal, catholic“ in: New Catholic encyclopedia 3 (2002), S. 392-393.

4 Ralph Lane Jr., „Catholic charismatic renewal“ in: The new religious consciousness (1976), S. 162-179. 
Formen der neuen Religiosität sein. Im Zusammenhang damit könnte man die Hypothese aufstellen, die zu Beginn der vorliegenden Forschung vorgeschlagen wurde, und zwar, dass die Konversion in der katholischen charismatischen Bewegung die religiöse Bekehrung zur Pfingstbewegung provozieren könnte. In diesem konkreten Fall hat sich die Forschung nur auf praktizierende katholische Gläubige konzentriert; nominelle Katholiken, die zu den pentekostalen und neopentekostalen Gemeinden konvertiert haben, wurden nicht mit einbezogen.

\section{Zum Problem der Typologie und Terminologie}

Bevor wir an die Analyse des vorliegenden Phänomens gehen, sollten wir erwähnen, was mit dem Begriff „katholische charismatische Bewegung“ gemeint ist. In der inländischen (nachsowjetischen) Literatur wird die katholische charismatische Bewegung nicht in vollem Umfang systematisch beschrieben, genauso wie diese Erscheinung selbst keine einheitlich strukturierte Definition kennt. Dabei halten die Vordenker der Bewegung auf der Ebene der höchsten Hierarchie (Kardinal L. J. Suenens) den Begriff selbst - „charismatisch“ - für nicht besonders treffend, weil er an und für sich keine präzise, abgrenzende Bedeutung hat: die ganze Kirche erscheint ja als ein Werk des Heiligen Geistes und hat folglich eine charismatische Struktur. ${ }^{5}$

Gleichzeitig, ausgehend von den von Adepten der Bewegung angewendeten Praktiken, setzt diese Definition eine enge Verbindung mit ekstatischen Zuständen voraus, die ein breites Spektrum der Erscheinungen umfassen; das lässt mehrere Ansätze zum Verstehen des Begriffs „Ekstase“ selbst vermuten, sowie eine Vielzahl von erarbeiteten Methoden zu dessen Untersuchung, was es schwer macht, den Begriff klar zu erfassen. ${ }^{6}$ Die Frage besteht insbesondere darin, ob das vorliegende Phänomen im Rahmen der katholischen Theologie $^{7}$ betrachtet oder aus der Position verschiedener wissenschaftlicher Ansätze ${ }^{8}$ analysiert werden sollte. Substantielle Bestimmungen der Ekstase sind ebenso unbefriedigend und unvollständig. Davon ausgehend schlägt die Mehrheit

\footnotetext{
5 Léon Joseph Suenens, A new Pentecost?, New York, 1975, S. 21-25.

${ }^{6}$ Diese Erscheinung wird in verschiedenen Quellen „Katholische charismatische Erneuerung“, „Katholische charismatische Bewegung“, „Erneuerung im Heiligen Geist“, „Bewegung der Wiedergeburt“, „katholische Pfingstbewegung“, „Pfingstbewegung in der katholischen Kirche" genannt. Auf solche Weise offenbart das Fehlen eines einheitlichen Begriffs für das genannte Phänomen das Vorhandensein von Diversifizierungsreferenzen in dieser Frage.

7 M. Frohlich, „Ecstasy“ in: New Catholic encyclopedia, 6 (2002), S. 60.

8 A. Sharma, Ecstasy, in: Encyclopedia of Religion, 4 (2005), S, 2678-2683; M. D. Beer, „Nature, causes and types of ecstasy“ in: Philosophy, Psychiatry \& Psychology, (4/2000), S. $311-315$.
} 
der Forscher eine korrelativ-dynamische Definition der Ekstase als eines ungewöhnlichen, nicht normalen Zustandes des Bewusstseins, oder, wie man heute üblicherweise sagt, als eines veränderten Zustandes des Bewusstseins, vor ${ }^{9}$. An diese Definition werden auch wir uns halten.

Der Versuch, die katholische charismatische Bewegung im Rahmen der Typologie von nicht traditionellen Formen der Religiosität zu klassifizieren, bereitet ebenso Schwierigkeiten. Unserer Meinung nach ist die Klassifizierung der nicht traditionellen Religiosität der Struktur nach, die von V. Martinovich aufgrund der Typologie der Kulte von Stark und Bainbridge ${ }^{10}$ entwickelt wurde, beachtenswert. Im Rahmen dieser Klassifikation kann man die katholische Pfingstbewegung dem kircheninneren Sektenwesen zurechnen, im Zusammenhang mit der „eigenwilligen Einführung in die Struktur der Kirche von ihr ursprünglich fremden religiösen Praktiken, Systemen der Glaubenslehren, Methoden der Arbeit “. ${ }^{11}$ Ausgehend von den von V. Martinovich beschriebenen Mechanismen der Zusammenwirkung dieser sektenähnlichen Gruppen mit den offiziellen Kirchenstrukturen, geben sich diese Organisationen Mühe, den Austritt von Trägern des kircheninneren Sektenwesens aus dem Schoß der Kirche nicht zuzulassen. Auf solche Weise zeigt der Versuch des systematischen Verständnisses der charismatischen Bewegung im Katholizismus - die Bestrebung, die „Bewegung der Wiedergeburt“ als eine Art geistliche Erneuerung darzustellen, die angesichts der Säkularisation der Kirche notwendig ist ${ }^{12}-$ die beschriebene Tendenz.

In der vorliegenden Arbeit wird vorgeschlagen, den Begriff „,katholische Pfingstbewegung“ als Synonym zum Begriff „katholische charismatische Bewegung“, dessen Verwendung in erster Linie durch die Verinnerlichung von pfingstbewegungseigenen Praktiken und Lehren in der katholischen Kirche bedingt ist, ${ }^{13} \mathrm{zu}$ gebrauchen.

9 V.E. Pashkovski, I. M.Zislin. „Религиозно-мистические состояния как психиатрическая проблема“ Социальная и клиническая психиатрия 15 (1/2005), S. 81-88.

10 Rodney Stark, William Sims Bainbridge, „Of churches, sects, and cults: Preliminary concepts for a theory of religious movements" in: Journal for the Scientific Study of Religion (1979), S. 117-131.

11 V. A. Martinovich, Нетрадиционная религиозность: возникновение и миграция, T.1, Минск 2015, S. 95-96.

12 Ibidem, S. 96

13 Zum Beispiel: Meredith B. McGuire, „Testimony as a commitment mechanism in Catholic Pentecostal prayer groups“ in: Journal for the Scientific Study of Religion (1977), S. 165-168; S. E. Ackerman, „The language of religious innovation: Spirit possession and exorcism in a Malaysian Catholic Pentecostal movement" in: Journal of Anthropological Research (1981), S. 90-100; Michael I. Harrison, „Sources of recruitment to Catholic pentecostalism“ 


\section{Katholische charismatische Gruppen in Weißrussland}

Leider fehlen inländische Arbeiten, die dieses Phänomen in unserem Land erforschen würden. So erwähnt O. Dyachenko ${ }^{14}$ das Vorhandensein der katholischen charismatischen Bewegung auf dem Territorium unseres Landes, aber eine Systemanalyse ihrer Dynamik führt er nicht durch. Dieser Umstand ist mit einer ganzen Reihe von Problemen zu erklären, mit denen der Forscher konfrontiert wird, wenn er versucht, die katholische Pfingstbewegung differenziert darzustellen: Mangel an empirischen Materialien, Geschlossenheit sowohl von offiziellen Kirchenstrukturen als auch von katholischen charismatischen Gruppen selbst, erschwerter Zugang zu der kritischen Literatur in dieser Frage usw. So ist die vorliegende Arbeit ein Novum, sowohl in inhaltlicher als auch in methodischer Beziehung.

Eine diachronisch-synchrone Analyse der katholischen charismatischen Bewegung in Weißrussland hat uns ermöglicht, die Geschichte ihrer Entstehung auf diesem Gebiet zu rekonstruieren. Das Aufkommen und die Verbreitung der katholischen Pfingstbewegung wurden erst nach der Wiederbelebung von Kirchenstrukturen der römisch-katholischen Kirche zu Beginn der 90er Jahre des XX. Jahrhunderts möglich. Ihre Verbreitung geschah durch polnische Priester und Mönche, die in nachsowjetischer Zeit nach Weißrussland gekommen waren, um das Wiederaufleben der katholischen Kirche zu unterstützen.

In Weißrussland gab es anfangs eine Konfrontation in den Beziehungen zwischen der katholischen charismatischen Bewegung und der institutionellen Kirche. Dies lässt sich am Beispiel der katholischen charismatischen Gruppe „Johannes der Täufer" aufzeigen, die im Jahre 1998 aus der römisch-katholischen Kirche ausgetreten ist und jetzt zur Vereinigten Kirche von Christen des Evangelischen Glaubens (OZHVE) gehört.

Den erwähnten Ereignissen ging die Ankunft des katholischen polnischen Volontärs Jaroslaw Lukasik als Missionar im Jahre 1993 in Minsk voraus. Seine Tätigkeit war in erster Linie auf die Evangelisierung der Jugend ausgerichtet. In demselben Jahr gründet er zum Zweck des Bibelstudiums die Gruppe „Johannes der Täufer“, die er ab jetzt leitet. Ein ausgeprägtes charismatisches Element beginnt die Gemeinde im Jahre 1997 zu gewinnen. Sein ausgeprägtes charismatischen Talent führt sogar dazu, dass der Akzent der

in: Journal for the Scientific Study of Religion (1974), S. 49-64; Ludovic Lado, Catholic Pentecostalism and the Paradoxes of Africanization: Processes of Localization in a Catholic Charismatic Movement in Cameroon, Vol. 37, Brill, 2009.

14 O. V. Dyachenko, „Пятидесятничество в Беларуси“, Могилев, 2003. 
Anbetung von Gott auf die Person von Jaroslaw Lukasik selbst verschoben wird, was von der Unterordnung unter seine Autorität begleitet wird, wobei sogar das Intimleben der Gruppenmitglieder reglementiert wird. 1998 erreichte die Konfrontation der Gemeinschaft mit der institutionellen Kirche ihren Höhepunkt. Infolgedessen hat sich ein Teil der charismatischen Gruppe von der katholischen Kirche abgespaltet und begann später, sich mit der Pfingstgemeinde zu identifizieren.

Aber der erwähnte Präzedenzfall soll als eine Ausnahme im Kontext des damaligen historisch-kulturellen Hintergrunds der neu auflebenden römisch-katholischen Kirche in Weißrussland betrachtet werden. Sie erlebte ihre Renaissance, aber wegen der Beschränktheit von Ressourcen und Kräften war sie nicht imstande, den Prozess der Aktivierung dieser Jugendgruppe zu kontrollieren. Die charismatische Einstellung der Gruppe war zu jener Zeit fremd und unverständlich, was zur Distanzierung der offiziellen Instanzen von der Tätigkeit und von den Praktiken dieser Gemeinde geführt hat.

In der modernen Phase ihrer Entwicklung ist die katholische charismatische Bewegung in zwei Hauptrichtungen aufgeteilt, die aber keine offizielle Unterstützung der Metropolie haben. „Schule des Christlichen Lebens und der Evangelisierung der Heiligen Maria von Nazareth, der Mutter der Kirche "15 vereinigt vorwiegend die Jugend in einem breiten Spektrum von Gebetsgruppen, Gemeinschaften, gesellschaftlichen Initiativen, Musik- und Internetprojekten.

Die Bewegung "Licht-Leben"16 ist in Struktureinheiten wie "Kostbare Perle“, „Oase“, „Familienoase“ organisiert. Sie wird von Priestern unterstützt und funktioniert vorwiegend in Gemeindegruppen mit dem Kirchenvorsteher an der Spitze. Sie steht in struktureller und ideologischer Verbindung mit der erstgenannten Strömung (die „Schule des Christlichen Lebens und der Evangelisierung"verwendet als Grundlagen ihrer Arbeit die Formationen und Stufen der Mitgliedschaft, die im Rahmen der Bewegung „Licht-Leben“erarbeitet wurden), aber sie besitzt eine bestimmte Autonomie, verwirklicht ihre Projekte und wirbt Adepten vorwiegend unter den Ehepaaren in mittlerem und fortgeschrittenem Alter.

Die katholische charismatische Bewegung in Weißrussland beschränkt sich nicht auf die genannten Strömungen, ihr schließen sich einzelne Gemeinden an, die den Prozess der Zentralisierung hin zu einer einheitlichen Struktur nicht durchgemacht haben und mit vereinzelten Gruppen präsent

15 Szkoła Życia Chrześcijańskiego i EwangelizacjiŚwiętej Maryi z Nazaretu Matki Kościoła, http://christschool.org.ua/index.php?lang=pl. abgerufen am 05. Mai 2015.

16 Рух «Святло-Жыцце», http://wsd.grodnensis.by/index.php?option=com_content\&vie $\mathrm{w}=$ article\&id=92\&Itemid=161\&lang=pl, abgerufen am 15. März 2014. 
sind. Solche charismatischen Gemeinden - „Mariä Licht“ und „Für den Gottvater" - haben keine einheitliche Verbindung mit den genannten Strömungen und offiziellen Kirchenstrukturen, aber sie suchen auch keine offene Konfrontation mit ihnen. In diesen Gruppen sind ekstatische Praktiken und mystische Erlebnisse stärker als in den zwei anderen Richtungen ausgeprägt.

Trotzdem können wir allgemeine Merkmale, die alle oben genannten charismatischen Gemeinden mit der römisch-katholischen Kirche gemeinsam haben, ausmachen. Der Akzent in den charismatischen Gebetsgruppen liegt vorwiegend auf der „Taufe durch den Heiligen Geist“ als einer eigenen geistlichen Erfahrung und auf "Seinen Gaben“, die in der Vorstellung von charismatischen Gruppen eine „außergewöhnliche Erscheinung der übernatürlichen Ordnung "17 sind. Dazu gehören auch sogenannte Epiphanien oder außerordentliche Gaben wie Glossolalie, „Fallen im Heiligen Geist“, Prophetie, die Fähigkeit der Unterscheidung der Geister, die Gabe der Heilung. Das Ritual ist schwach ausgeprägt und geordnet, es enthält spontane Elemente, was uns erlaubt, die Tendenz des Übergangs von einem klar definierten Ritus, wie es ihn in der katholischen Kirche gibt, zu vielfältiger entwickelten rituellen Formen zu erkennen.

Als ein Verbindungselement im Glaubenssystem der genannten Gemeinden treten die „Offenbarungen“ auf, die ihre Führer von der Jungfrau Maria, von Jesus Christus, von Heiligen und Engeln erhalten haben (im Falle der Gruppe „Für den Gottvater“ gehen die Offenbarungen und Prophezeiungen auch von der ersten Person der Dreifaltigkeit aus). Das nächste Merkmal liegt auf dem Gebiet der religiösen Ideologie; für diese Ideologie sind apokalyptische Motive der Wiederkunft Christi und der Leiden vor dem Weltende, und als Folge davon die in den Gruppen vorherrschende Überzeugung, dass der Anhänger der Bewegung bereits gerettet ist, kennzeichnend. Die Behauptung eines unmittelbaren Wirkens des Teufels hat ebenfalls eine zentrale Bedeutung für solche charismatischen Gemeinden. Demzufolge sind in den Gruppen die Methoden der massenhaften Vertreibung der bösen Geister und die Fürbitten, die ihnen widerstehen sollen, sehr verbreitet. Die „bösen Kräfte" werden manchmal mit den Personen identifiziert, die charismatische Praktiken kritisieren, oder sie werden in den Problemen gesehen, mit denen nicht nur die Gruppe, sondern auch die ganze Konfession konfrontiert wird (zum Beispiel die Propagierung von empfängnisverhütenden Mitteln). Die Krankheiten und persönlichen Schwierigkeiten der Mitglieder der Bewegung werden ebenso dem Einfluss des Teufels zugeschrieben.

17 G. T. Montague, "Charismatic prayer“ in: New Catholic encyclopedia, 3 (2002), S. 391392. 
Für die charismatische Bewegung ist die autoritäre Rolle des Führers sowohl als Gruppenoberhaupt als auch als Führer der ganzen Richtung charakteristisch. Und wie aus der religiösen Analyse von charismatischen Gemeinden erkennbar ist, führt die Aufstellung von Regeln seitens des Gruppenführers zwecks Anleitung zum rechten Benehmen der Adepten zur Verminderung ihres Charismas. Im Zusammenhang mit der Tendenz zur Kontrolle, die in den zu erforschenden Gemeinschaften festgestellt wird, und mit der vergleichenden Analyse der charismatischen Bewegung in Polen und in der Ukraine, kann man behaupten, dass das charismatische Element in der „Bewegung der Wiedergeburt" auf dem Gebiet von Weißrussland bedeutend schwächer ausgeprägt ist.

\section{Schlussfolgerung}

Statt einer Schlussfolgerung möchten wir eine relative Typologie von weißrussischen katholischen charismatischen Gruppen vorschlagen, deren Kriterium das Ausmaß sein kann, in dem die Führer den Mitgliedern und Beobachtern erlauben, in den Gebetsversammlungen zu sprechen oder deren Verlauf zu beeinflussen: ${ }^{18}$

Geschlossene Gruppen kennzeichnen sich dadurch aus, dass die Gebetstreffen von den Nichtmitgliedern der Gemeinde nicht besucht werden können. Sie sind nicht auf die externe Welt und die Missionstätigkeit ausgerichtet, sie versuchen die Kontrolle über das Benehmen, die Gedanken, Emotionen und hereinkommende Informationen auszuüben (es gibt eine Sammlung von stillen Regeln, unter deren Einfluss alle Lebenssphären des Individuums geraten), was zu ihrer Entcharismatisierung führt; zu diesem Typus gehört die Gruppe des „Gottvaters“.

Halbgeschlossene Gebetsgemeinschaften üben die Missionstätigkeit aktiv aus und bleiben für externe Kontakte offen; die Neubekehrten können am Gebetstreffen aktiv teilnehmen und dessen Verlauf beeinflussen, aber diese Charakteristiken sind statisch; in den Frühlings- und Sommermonaten schließen sich die Gemeinden von der externen Umgebung ab und stellen die Missionstätigkeit ein, im Zusammenhang mit dem Zufluss von neuen Mitgliedern und mit der Notwendigkeit, sie zu „lehren“. Dieser Typus der Gruppen ist für die Kirchengebetsgemeinden charakteristisch; außerdem gehören auch die Gebetsgruppen dazu, die sich nach dem Geschlechtsprinzip unterscheiden.

18 Als Grundlage wird die Typologie verwendet, die im Artikel: P. Halama, J. Halamová, „Process of religious conversion“" vorgeschlagen wurde. 
Maryna Kazmiruk

Offene Gruppen sind aktiv, auf die externe Welt ausgerichtet, daran können alle Interessenten, unabhängig von ihrer Konfession, frei teilnehmen; sie zeichnen sich durch Mobilität aus, dabei sind der größte Teil der Besucher dieser Gruppe die Vertreter der Pfingstbewegungen.

Es muss darauf hingewiesen werden, dass die aufgeführte Typologie vorläufig ist und einer weiteren Ausbesserung und Nachbearbeitung bedarf. $\mathrm{Zu}$ ihren Nachteilen gehört die Tatsache, dass das erarbeitete Modell in erster Linie am Beispiel von charismatischen Jugendgruppen, in denen das ekstatische Element stärker ausgeprägt ist, aufgebaut wurde. 